Journal of Case Reports 2018;8(4):236-239

\title{
Simultaneous Replantation at Wrist Joint and Revascularization at Elbow Joint in Peripheral Hospital Setup
}

\author{
Prashant Moon, Meera Pandey \\ Department of Plastic and Reconstructive Surgery, Krishna Hospital and Research Centre, Haldwani, Uttarakhand, India.
}

\author{
Corresponding Author: \\ Dr. Prashant Moon \\ Email: prashantemoon@gmail.com
}

This is an Open Access article distributed under the terms of the Creative Commons Attribution License (creativecommons.org/ licenses/by/3.0).

Received

Accepted

Published

July 5, 2018

September 17, 2018

October 15,2018

\begin{abstract}
Background: Replantation and revascularization surgery is a procedure which requires adequate training, good knowledge of microsurgical technique and proper infrastructure and instrument. In India replantation is most of the time performed in tertiary centre which are having good infrastructure and team of plastic surgeons well trained in microsurgical procedure. Case Report: We hereby report simultaneous replantation at wrist level and revascularization at elbow level in a 45 year old female factory worker in a Kumaoun region of India. Conclusion: Decision of reimplantation and revascularization in patient is based upon the patient's age, occupation, socio-economic status and expected return of function after surgery.
\end{abstract}

Keywords: Elbow Joint, Humans, Microsurgery, Replantation, Wrist Joint.

\section{Introduction}

In India, replantation and revascularization procedures are most of the time performed in highly trained tertiary care setup. There is team of plastic surgeons which are available round the clock to perform the surgery. Patients who are living far away from these setup rarely get benefitted from this facility. Facility for replantation and revascularization should also be available at peripheral level so that chances of limb salvage can be maximized [1-3]. There should be adequate training of plastic surgeon for performing micro-vascular surgery so that they can perform replantation procedure timely to salvage the limb. We are reporting a case of simultaneous replantation at wrist level and revascularization at elbow level in factory worker in a Kumaoun region of India.

\section{Case Report}

A 45 year old female had a history of amputation of right hand at wrist level and deep cut laceration at elbow level due to hand caught in atta (wheat flour) machine while working in factory. Patient had come to the hospital after two hours of injury. Initial resuscitation was done in emergency department. Her pulse was 82 beats per minute, blood pressure was $110 / 70 \mathrm{~mm} \mathrm{Hg}$. hemoglobin concentration was $11.7 \mathrm{gm} / \mathrm{L}$. Examination showed bones, nerves, tendons, and vessels of his affected limbs were avulsed at right wrist [Fig.1,2]. There was deep lacerated wound over right elbow joint [Fig.3]. Pre-operative X-ray was showing normal right elbow joint [Fig.4]. The wounds were contaminated with atta (wheat flour). There was no other additional injury over body. There was a torrential bleeding from elbow wound for which compression and elevation was given. Amputed part was covered in wet saline and stored in sterile polythene bag in ice saline slurry.

Patient was shifted to operation theatre and general anaesthesia was given. Tourniquet was applied to upper arm to prevent further blood loss. Painting and draping was done. Exploration 


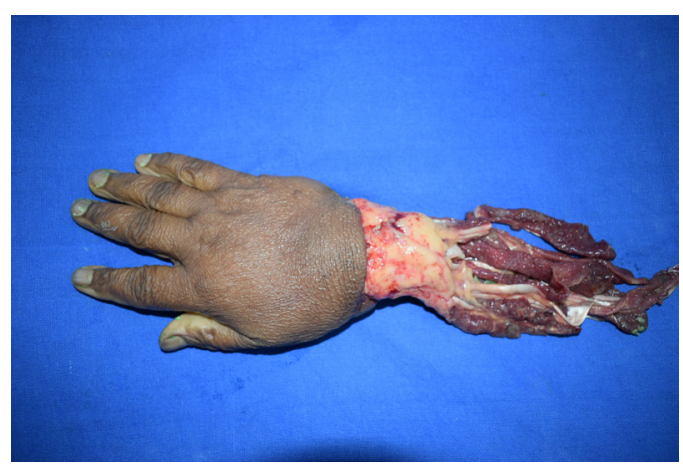

Fig.1: Avulsion amputaition at wrist level, dorsal view.

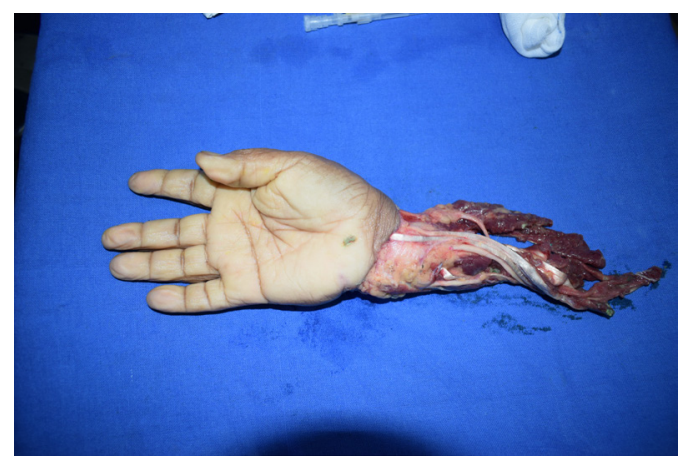

Fig.2: Avulsion amputaion at wrist level, volar view.

of wound was done suggestive of amputation of hand at wrist level with avulsion of tendons and arteries. There was also a deep cut wound over elbow level with partial tear of brachial artery. Preparation of proximal and distal part was done. Trans-carpal fixation of wrist was done with radius and ulna with two $2 \mathrm{~mm} \mathrm{~K}$ wire from second and fourth metacarpal bone. Repair of brachial artery was done at elbow. Repair of radial artery, ulnar artery, three vena commitants, and three dorsal vein repair were done. All flexors were identified and repaired at the level of tendon-muscle junction. Extensors were identified and repair done. Median nerve, ulnar nerve, bicep brachii tendon repair was done [Fig. 5]. Skin closure done and straight splint was given. Patient was shifted to intensive care unit for further post-operative management. She was managed with daily regular loose dressing. Skin temperature, venous congestion and capillary

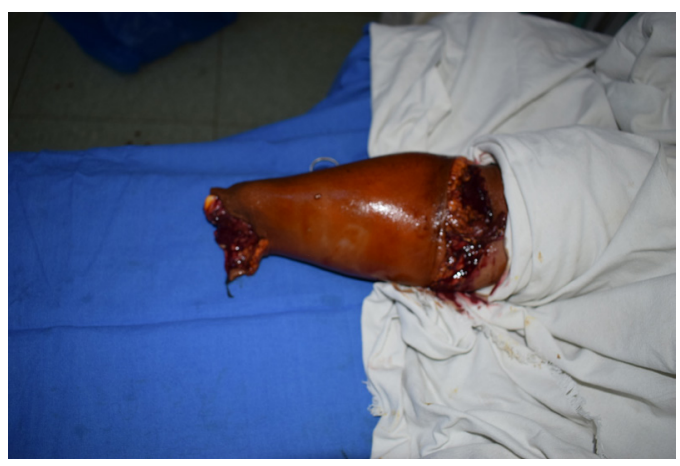

Fig.3: Deep laceration with brachial artery injury at elbow level.

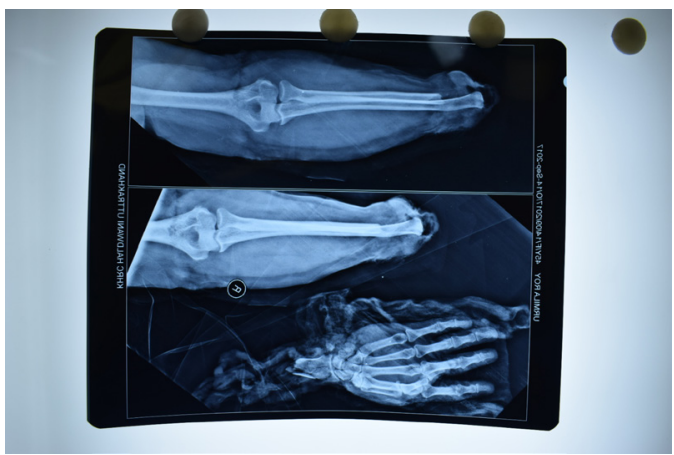

Fig.4: Pre-operative skiagram of right upper limb.

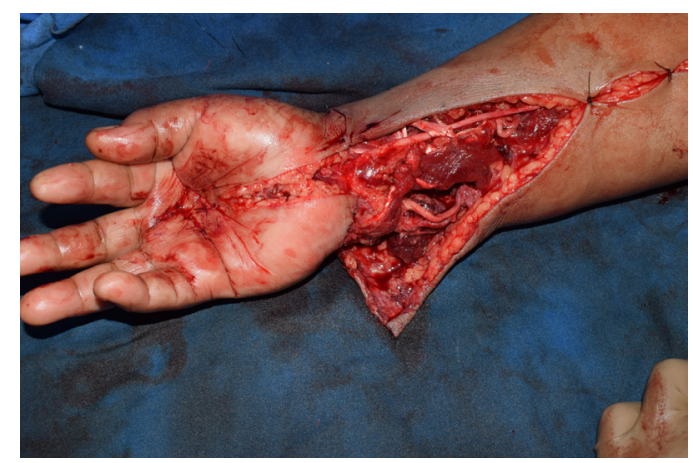

Fig.5: Intra-operative photograph.

refill, and pulse oximetry were closely monitored. Her post-operative course was uneventful except marginal necrosis of skin over flexor aspect of forearm. Patient was kept on intravenous heparin for five days. Patient was discharged from hospital on $14^{\text {th }}$ post-operative day. Her physiotherapy was started after three weeks of injury [Fig. 6]. 


\section{Discussion}

Replantation at multiple levels, avulsion amputation, and amputation at proximal level remains challenging surgery to the microvascular surgeon. These conditions are often considered as a relative contraindication for replantation. Many studies have shown that percentage of successful replantation was more in case of clean cut amputations, young patients, and with early presentations [1]. Decision of reimplantation and revascularization in such patient should be individualized based upon the patient's age, occupation, socio-economic status and expected return of function after surgery.

Even though replantation surgery has now become a routine procedure, it remains delicate and demanding surgery, requiring adequate training and expertise in microsurgical techniques. Although replantation procedures have been simplified, a second surgical team can save valuable surgical time by debridement and identifying the vessels in the amputated part, harvesting venous grafts, and performing bone fixation or tendon repair among other things, while the chief surgeon focuses on revascularization. To carry out two prolonged microvascular procedures simultaneously is extremely difficult. Manpower, both skilled and unskilled in addition to the equipment and resources need to be doubled. Sequential vascular repair is necessary when only a single microscope is available. In peripheral setup it is very difficult to get two surgical teams for replantation surgery [3].

Replantation of multiple digits and hand remains a daunting challenge that presents especially if it is avulsion-crush injury. The hand crushing, pulling and rotating forces are responsible for the most severe injuries to amputated parts and are often contraindications for replantation [4]. When patient with amputation came to the casualty initial evaluation for patient's vitals and associated injury should be done before checking condition of the amputed part. Amputated part is wrapped in wet

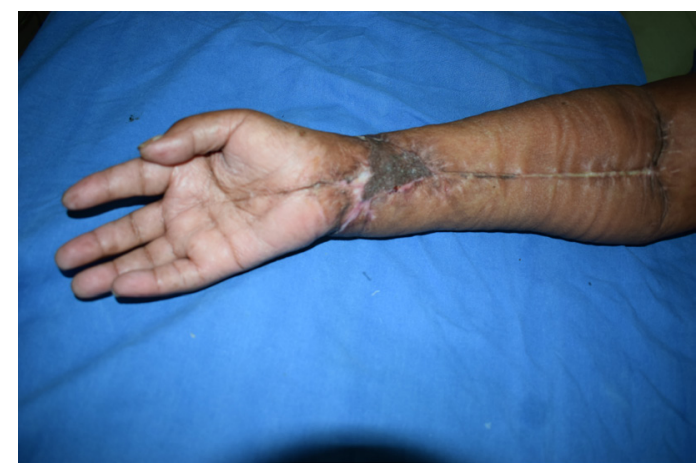

Fig.6: Post-operative, volar view.

saline gauze dressing and kept in refrigerator. Do not keep amputated part in direct contact with ice. Amputation stump should be closed with dressing and kept elevated to control further blood loss from amputation stump [5,6]. Amputation at wrist and above should be replanted within six hours of warm ischemia and 12 hours of cold ischemia.

Patient and relative should be well informed regarding replantation procedure, risk of complication, expenditure, chances of amputation if surgery fails, and expected functional outcome after surgery. Post-operative management of patient includes monitoring of patients vitals, hourly monitoring of hand, clinically as well as with pulse-oximetry. Patient should be kept in intensive care unit for at least 48 to 72 hours [4]. Intravenous heparin infusion should be given in patient with history of avulsion amputation [6].

Physiotherapy and occupation therapy is essential component of any replantation procedure [5]. Patient should be put on physiotherapy after healing of wound. Successful replantation is considered as a return of blood circulation and patients function [2]. Functional outcome of replanted hands and fingers will never equal that of the normal healthy counterpart, replantation has major functional, cosmetic and psychological benefits [7].

\section{Conclusion}

Decision of reimplantation and revascularization in patient is based upon the patient's age, occupation, 
socio-economic status and expected return of function after surgery.

Contributors: PM: manuscript editing and critical inputs into the manuscript; MP: manuscript drafting and literature search. PM will act as guarantor. Both authors approved the final version of this manuscript.

Funding: None; Competing interests: None stated.

\section{References}

1. Kamarul T, Mansor A, Robson N, Albusaidi SHH, Abdulrazzaq M Suhaeb, et al. Replantation and revascularization of amputated upper limb appendages outcome and predicting the factors influencing the success rates of these procedures in a tertiary hospital: An 8-year retrospective, cross-sectional study. Journal of Orthopaedic Surgery. 2018;26:1.

2. Beaton, DE, Katz, JN, Fossel, AH. Measuring the whole or the parts? Validity, reliability, and responsiveness of the disabilities of the arm, shoulder and hand outcome measure in different regions of the upper extremity. J Hand Ther. 2001;14:128-146.

3. NayakBB, Mohanty N, PatnaikAP, BalPK. Consideration for double hand replantation in a resources-constricted healthcare facility. Indian J Plast Surg. 2016:49:81-85.

4. Gupta A, Pawar HS, Mehta R, Goyal S. Toka machine injury: Replantation left arm in a 5 year old. Apollo Medicine. 2015:12:50-53.

5. Kadam D. Limb salvages surgery. Indian J Plast Surg. 2013:46:265-274.

6. Nanda V, Jacob J, Alsafy T, Punnoose T; Sudhakar VR, Iyasere G. Replantation of an amputated hand: a rare case report and acknowledgement of a multidisciplinary team input. Oman Med J. 2011:26(4):2780282.

7. Salah MM, Khalid KN. Replantation of multiple digits and hand amputations: four case reports. Cases Journal. 2008;1:266. 